\title{
On existence and uniqueness of solutions of nonlocal problems for hyperbolic differential-functional equations in two independent variables
}

\author{
by Tomasz CzŁapiński (Gdańsk)
}

\begin{abstract}
We seek for classical solutions to hyperbolic nonlinear partial differentialfunctional equations of the second order. We give two theorems on existence and uniqueness for problems with nonlocal conditions in bounded and unbounded domains.
\end{abstract}

1. Introduction. If $X, Y$ are any metric spaces then we denote by $C(X ; Y)$ the set of all continuous functions from $X$ to $Y$. Let $\mathbb{R}_{+}=[0, \infty)$, $a_{0}, b_{0} \in \mathbb{R}_{+}$and $a>0, b>0$. We put $\Omega=[0, a] \times[0, b], \widetilde{\Omega}=\left[-a_{0}, a\right] \times$ $\left[-b_{0}, b\right], \Omega_{0}=\left[-a_{0}, a\right] \times\left[-b_{0}, b\right] \backslash(0, a] \times(0, b]$ and $B=\left[-a_{0}, 0\right] \times\left[-b_{0}, 0\right]$. If $z: \widetilde{\Omega} \rightarrow \mathbb{R}$ then for any $(x, y) \in \Omega$ we define a function $z_{(x, y)}: B \rightarrow \mathbb{R}$ by $z_{(x, y)}(s, t)=z(x+s, y+t),(s, t) \in B$.

In this paper we will deal with the following nonlinear hyperbolic differential-functional equation:

$$
D_{x y} u(x, y)=f\left(x, y, u_{(x, y)}, D_{x} u(x, y), D_{y} u(x, y)\right), \quad(x, y) \in \Omega,
$$

where $f: \Omega \times C(B ; \mathbb{R}) \times \mathbb{R}^{2} \rightarrow \mathbb{R}$, together with the nonlocal conditions

$$
\begin{array}{ll}
u_{(x, 0)}+\sum_{i=1}^{n}\left(h_{i}\right)_{(x, 0)} u_{\left(x, b_{i}\right)}=\phi_{(x, 0)}, & x \in[0, a], \\
u_{(0, y)}+\sum_{j=1}^{m}\left(g_{j}\right)_{(0, y)} u_{\left(a_{j}, y\right)}=\phi_{(0, y)}, & y \in[0, b],
\end{array}
$$

where $\phi: \Omega_{0} \rightarrow \mathbb{R}, h_{i}:\left[-a_{0}, a\right] \times\left[-b_{0}, 0\right] \rightarrow \mathbb{R}, i=1, \ldots, m, g_{j}:\left[-a_{0}, 0\right] \times$ $\left[-b_{0}, b\right] \rightarrow \mathbb{R}, j=1, \ldots, m$, and $a_{j}, j=1, \ldots, m, b_{i}, i=1, \ldots, n$, are finite numbers such that $0<a_{1}<\ldots<a_{m} \leq a, 0<b_{1}<\ldots<b_{n} \leq b$. The

1991 Mathematics Subject Classification: 35L10, 35L20, 35R10.

Key words and phrases: differential-functional equations, nonlinear hyperbolic problems, nonlocal conditions. 
nonlocal conditions (2), (3) may also be written in the form

$$
\begin{aligned}
u(x, y)+\sum_{i=1}^{n} h_{i}(x, y) u\left(x, b_{i}+y\right) & =\phi(x, y), \\
(x, y) & \in\left[-a_{0}, a\right] \times\left[-b_{0}, 0\right], \\
u(x, y)+\sum_{j=1}^{m} g_{j}(x, y) u\left(a_{j}+x, y\right) & =\phi(x, y), \\
(x, y) & \in\left[-a_{0}, 0\right] \times\left[-b_{0}, b\right] .
\end{aligned}
$$

R e mark 1. Note that the domains on which the nonlocal conditions $\left(2^{\prime}\right),\left(3^{\prime}\right)$ are considered overlap. To ensure compatibility we need additional assumptions on the functions $h_{i}, g_{j}$. In the sequel we will assume that $h_{i}(x, y)=g_{j}(x, y)=0$ for $(x, y) \in B, i=1, \ldots, n, j=1, \ldots, m$.

If $a_{0}=0, b_{0}=0$ then the differential-functional problem (1)-(3) reduces to a differential nonlocal problem. A problem of this type, in which $g_{j}=$ $0, j=1, \ldots, m$, was considered by L. Byszewski [2]. The nonlocal conditions were introduced for the first time by J. Chabrowski [3] in the study of linear parabolic problems. Conditions of this type can be applied in the theory of elasticity with better results than the initial or Darboux conditions. Nonlinear differential problems of parabolic type with nonlocal inequalities together with their physical interpretation were considered by L. Byszewski [1]. His results concerning nonlocal problems are generalizations of those given in [4], [7] since in case $h_{i}=g_{j}=0, i=1, \ldots, n, j=1, \ldots, m$, the nonlocal conditions reduce to the classical Darboux conditions (see also [6], $[8])$.

In this paper we consider nonlocal problems for the differential-functional equation (1), i.e. for the equation in which the right hand side is an operator on the function space $C(B ; \mathbb{R})$ with respect to the third variable. The Darboux problem for nonlinear hyperbolic differential-functional equations in a Banach space was studied in [5] with the use of the Kuratowski $\alpha$-measure of noncompactness. In this paper we give a theorem on existence and uniqueness of classical solutions of the problem (1)-(3). We also give an analogous theorem for a problem on an unbounded domain, with $\Omega,[0, a]$ and $[0, b]$ replaced by $\mathbb{R}_{+}^{2}, \mathbb{R}_{+}$and $\mathbb{R}_{+}$, respectively. The proof is based on the Banach fixed-point theorem and it is close to that given in [2].

2. Theorems. By $C^{1}(\Omega ; \mathbb{R})$ we denote the space of all functions $u \in$ $C(\Omega ; \mathbb{R})$ which have continuous derivatives $D_{x} u, D_{y} u$ on $\Omega$. Let $U$ be the set of all functions $u \in C(\widetilde{\Omega} ; \mathbb{R})$ which are $C^{1}$ on $\Omega$ and such that the mixed derivative $D_{x y} u$ exists and is continuous on $\Omega$, and let $\|\cdot\|$ denote the usual supremum norm in $C(B ; \mathbb{R})$. 
Theorem 1. Suppose that

(i) $f \in C\left(\Omega \times C(B, \mathbb{R}) \times \mathbb{R}^{2} ; \mathbb{R}\right)$ and there is a nonnegative constant $L$ such that for all $(x, y) \in \Omega, z, \bar{z} \in C(B ; \mathbb{R}), p, \bar{p}, q, \bar{q} \in \mathbb{R}$, we have

$$
|f(x, y, z, p, q)-f(x, y, \bar{z}, \bar{p}, \bar{q})| \leq L\{\|z-\bar{z}\|+|p-\bar{p}|+|q-\bar{q}|\} ;
$$

(ii) $\phi \in C\left(\Omega_{0} ; \mathbb{R}\right)$ and $\phi(0, \cdot) \in C^{1}([0, a] ; \mathbb{R}), \phi(\cdot, 0) \in C^{1}([0, b] ; \mathbb{R})$;

(iii) $h_{i} \in C^{1}\left(\left[-a_{0}, a\right] \times\left[-b_{0}, 0\right] ; \mathbb{R}\right), i=1, \ldots, n, g_{j} \in C^{1}\left(\left[-a_{0}, 0\right] \times\right.$ $\left.\left[-b_{0}, b\right] ; \mathbb{R}\right), j=1, \ldots, m$, and

(5) $\quad h_{i}(x, y)=0, \quad g_{j}(x, y)=0, \quad(x, y) \in B, i=1, \ldots, n, j=1, \ldots, m$;

(iv) $K, M$ are nonnegative constants such that for all $(x, y) \in \Omega, i=$ $1, \ldots, n, j=1, \ldots, m$, we have

$$
\begin{aligned}
& \left|h_{i}(x, y)\right| \leq N, \quad\left|D_{x} h_{i}(x, y)\right| \leq N, \\
& \left|g_{j}(x, y)\right| \leq M, \quad\left|D_{y} g_{j}(x, y)\right| \leq M ;
\end{aligned}
$$

(v) there is a positive constant $C$ such that

$$
\frac{L(2 C+1)}{C^{2}}+2 n N e^{C b_{n}}+2 m M e^{C a_{m}}<1 .
$$

Then there is a unique solution of the problem (1)-(3) in the class $U$.

Proof. Let $\widehat{U}$ be the set of all functions $u \in C(\widetilde{\Omega} ; \mathbb{R})$ which are $C^{1}$ on $\Omega$. In $\widehat{U}$ we introduce the norm

$$
\|u\|_{C}=\|u\|_{C, 0}+\|u\|_{C, 1}+\|u\|_{C, 2}
$$

where

$$
\begin{aligned}
\|u\|_{C, 0} & =\sup _{(x, y) \in \widetilde{\Omega}} e^{-C(x+y)}|u(x, y)|, \\
\|u\|_{C, 1} & =\sup _{(x, y) \in \Omega} e^{-C(x+y)}\left|D_{x} u(x, y)\right|, \\
\|u\|_{C, 2} & =\sup _{(x, y) \in \Omega} e^{-C(x+y)}\left|D_{y} u(x, y)\right| .
\end{aligned}
$$

We define the following operator on $\widehat{U}$ :

(8)

$$
\begin{aligned}
(T u)(x, y)= & \phi(x, 0)+\phi(0, y)-\phi(0,0) \\
& -\sum_{i=1}^{n} h_{i}(x, 0) u\left(x, b_{i}\right)-\sum_{j=1}^{m} g_{j}(0, y) u\left(a_{j}, y\right) \\
& +\int_{0}^{x y} \int_{0}^{x} f\left(s, t, u_{(s, t)}, D_{x} u(s, t), D_{y} u(s, t)\right) d t d s,
\end{aligned}
$$

$$
(x, y) \in \Omega,
$$




$$
\begin{aligned}
(T u)(x, y)=\phi(x, y)-\sum_{i=1}^{n} h_{i}(x, y) u\left(x, b_{i}+y\right) & \\
(x, y) & \in\left[-a_{0}, a\right] \times\left[-b_{0}, 0\right), \\
(T u)(x, y)=\phi(x, y)-\sum_{j=1}^{m} g_{j}(x, y) u\left(a_{j}+x, y\right), & \\
(x, y) & \in\left[-a_{0}, 0\right) \times\left[-b_{0}, b\right] .
\end{aligned}
$$

The above definition is correct even though the domains in (9) and (10) overlap. Indeed, if $(x, y) \in B$ then by $(5)$ we see that $(T u)(x, y)=\phi(x, y)$ in (9) as well as in (10).

It is easy to see that if $u \in \widehat{U}$ then $T u$ is continuous on $\widetilde{\Omega} \backslash \Omega$ and $C^{1}$ on $\Omega$. The continuity of $T u$ on $\widetilde{\Omega}$, i.e. the continuity on $\{0\} \times[0, b] \cup[0, a] \times\{0\}$, follows immediately from (5). Therefore, $T$ maps $\widehat{U}$ into itself.

If $u \in U$ is a solution of the problem (1)-(3) then integrating (1) on $[0, x] \times[0, y]$ and making use of $(2),(3)$ we find that $u$ is a fixed point of $T$. Conversely, if $u \in \widehat{U}$ is a fixed point of $T$ then from (8) we see that $u$ has a continuous derivative $D_{x y} u$ on $\Omega$, and that equation (1) holds true. Morover, (2), (3) follow immediately from (9), (10). Thus we will seek for a fixed point of the operator $T$.

If $u, \bar{u} \in \widehat{U}$ then by (4), (6), (8)-(10), we have

$$
\begin{aligned}
& |(T u)(x, y)-(T \bar{u})(x, y)| \\
& \leq L \int_{0}^{x} \int_{0}^{y}\left[\left\|u_{(s, t)}-\bar{u}_{(s, t)}\right\|\right. \\
& \left.\quad+\left|D_{x} u(s, t)-D_{x} \bar{u}(s, t)\right|+\left|D_{y} u(s, t)-D_{y} \bar{u}(s, t)\right|\right] d t d s \\
& \quad+\sum_{i=1}^{n}\left|h_{i}(x, 0)\right| \cdot\left|u\left(x, b_{i}\right)-\bar{u}\left(x, b_{i}\right)\right|+\sum_{j=1}^{m}\left|g_{j}(0, y)\right| \cdot\left|u\left(a_{j}, y\right)-\bar{u}\left(a_{j}, y\right)\right| \\
& \leq L \int_{0}^{x}\left[\|u-\bar{u}\|_{C, 0}+\|u-\bar{u}\|_{C, 1}+\|u-\bar{u}\|_{C, 2}\right] e^{C(s+t)} d t d s \\
& \quad+\sum_{i=1}^{n} N e^{C\left(b_{i}+x+y\right)}\|u-\bar{u}\|_{C, 0}+\sum_{j=1}^{m} M e^{C\left(a_{j}+x+y\right)}\|u-\bar{u}\|_{C, 0} \\
& \leq e^{C(x+y)} \\
& \quad \times\left\{\frac{L}{C^{2}}\|u-\bar{u}\|_{C}+\left[n N e^{C b_{n}}+m M e^{C a_{m}}\right]\|u-\bar{u}\|_{C, 0}\right\} \quad \text { for }(x, y) \in \Omega,
\end{aligned}
$$




$$
\begin{aligned}
& |(T u)(x, y)-(T \bar{u})(x, y)| \\
& \quad \leq \sum_{i=1}^{n}\left|h_{i}(x, y)\right| \cdot\left|u\left(x, b_{i}+y\right)-\bar{u}\left(x, b_{i}+y\right)\right| \\
& \quad \leq \sum_{i=1}^{n} N e^{C\left(b_{i}+x+y\right)}\|u-\bar{u}\|_{C, 0} \\
& \quad \leq e^{C(x+y)} n N e^{C b_{n}}\|u-\bar{u}\|_{C, 0} \quad \text { for }(x, y) \in\left[-a_{0}, a\right] \times\left[-b_{0}, 0\right],
\end{aligned}
$$

and

$$
\begin{aligned}
& |(T u)(x, y)-(T \bar{u})(x, y)| \\
& \quad \leq \sum_{j=1}^{m}\left|g_{j}(x, y)\right| \cdot\left|u\left(a_{j}+x, y\right)-\bar{u}\left(a_{j}+x, y\right)\right| \\
& \quad \leq \sum_{j=1}^{m} M e^{C\left(a_{j}+x+y\right)}\|u-\bar{u}\|_{C, 0} \\
& \quad \leq e^{C(x+y)} m M e^{C a_{m}}\|u-\bar{u}\|_{C, 0} \quad \text { for }(x, y) \in\left[-a_{0}, 0\right] \times\left[-b_{0}, b\right] .
\end{aligned}
$$

From the above estimates we get

(11) $\quad\|T u-T \bar{u}\|_{C, 0} \leq \frac{L}{C^{2}}\|u-\bar{u}\|_{C}+\left[n N e^{C b_{n}}+m M e^{C a_{m}}\right]\|u-\bar{u}\|_{C, 0}$.

In the same manner from (4), (6), (8), and from the estimates

$$
\begin{aligned}
\mid D_{x}(T u)(x, y) & -D_{x}(T \bar{u})(x, y) \mid \\
\leq & L \int_{0}^{y}\left[\| u_{(x, t)}-\bar{u}_{(x, t)}||\right. \\
& \left.+\left|D_{x} u(x, t)-D_{x} \bar{u}(x, t)\right|+\left|D_{y} u(x, t)-D_{y} \bar{u}(x, t)\right|\right] d t \\
& +\sum_{i=1}^{n}\left|D_{x} h_{i}(x, 0)\right| \cdot\left|u\left(x, b_{i}\right)-\bar{u}\left(x, b_{i}\right)\right| \\
& +\sum_{i=1}^{n}\left|h_{i}(x, 0)\right| \cdot\left|D_{x} u\left(x, b_{i}\right)-D_{x} \bar{u}\left(x, b_{i}\right)\right|
\end{aligned}
$$

and

$$
\begin{aligned}
& \left|D_{y}(T u)(x, y)-D_{y}(T \bar{u})(x, y)\right| \\
& \quad \leq L \int_{0}^{x}\left[\left\|u_{(s, y)}-\bar{u}_{(s, y)}\right\|\right. \\
& \left.\quad+\left|D_{x} u(s, y)-D_{x} \bar{u}(s, y)\right|+\left|D_{y} u(s, y)-D_{y} \bar{u}(s, y)\right|\right] d s
\end{aligned}
$$




$$
\begin{aligned}
& +\sum_{j=1}^{m}\left|D_{y} g_{j}(0, y)\right| \cdot\left|u\left(a_{j}, y\right)-\bar{u}\left(a_{j}, y\right)\right| \\
& +\sum_{j=1}^{m}\left|g_{j}(0, y)\right| \cdot\left|D_{y} u\left(a_{i}, y\right)-D_{y} \bar{u}\left(a_{i}, y\right)\right|,
\end{aligned}
$$

which hold true for $(x, y) \in \Omega$, we get

$$
\begin{aligned}
\|T u-T \bar{u}\|_{C, 1} \leq & \frac{L}{C}\|u-\bar{u}\|_{C}+n N e^{C b_{n}}\|u-\bar{u}\|_{C, 0} \\
& +n N e^{C b_{n}}\|u-\bar{u}\|_{C, 1}, \\
\|T u-T \bar{u}\|_{C, 2} \leq & \frac{L}{C}\|u-\bar{u}\|_{C}+m M e^{C a_{m}}\|u-\bar{u}\|_{C, 0} \\
& +m M e^{C a_{m}}\|u-\bar{u}\|_{C, 2} .
\end{aligned}
$$

Therefore, by (11)-(13), we get

$$
\|T u-T \bar{u}\|_{C} \leq\left\{\frac{L(2 C+1)}{C^{2}}+2 n N e^{C b_{n}}+2 m M e^{C a_{n}}\right\}\|u-\bar{u}\|_{C} .
$$

This together with (7) yields that $T$ is a contraction. By the Banach fixed point theorem we conclude that $T$ has a unique fixed point in $\widehat{U}$, which completes the proof of Theorem 1 .

Remark 2. The proof of Theorem 1 still goes when the condition (iii) is replaced by: For all $i=1, \ldots, n, j=1, \ldots, m$, we have

(a) $h_{i} \in C\left(\left[-a_{0}, a\right] \times\left[-b_{0}, 0\right] ; \mathbb{R}\right), g_{j} \in C\left(\left[-a_{0}, 0\right] \times\left[-b_{0}, b\right] ; \mathbb{R}\right)$;

(b) $h_{i}$ is $C^{1}$ on $[0, a] \times\left[-b_{0}, 0\right]$ and $g_{j}$ is $C^{1}$ on $\left[-a_{0}, 0\right] \times[0, b]$;

(c) $h_{i}(x, y)=g_{j}(x, y)=0$, for $(x, y) \in B$.

Now, we consider the problem (1)-(3) with $\Omega,[0, a]$ and $[0, b]$ replaced by $\mathbb{R}_{+}^{2}, \mathbb{R}_{+}$and $\mathbb{R}_{+}$, respectively. Consequently, in the definitions of the spaces $U, \widehat{U}$, of the operator $T$, and of the norm $\|\cdot\|_{C}$, we replace $\Omega, \widetilde{\Omega}$ and $\Omega_{0}$ by $\mathbb{R}_{+}^{2},\left[-a_{0}, \infty\right) \times\left[-b_{0}, \infty\right)$ and $\left[-a_{0}, \infty\right) \times\left[-b_{0}, \infty\right) \backslash(0, \infty)^{2}$, respectively.

Theorem 2. Suppose that

(i) $f \in C\left(\mathbb{R}_{+}^{2} \times C(B ; \mathbb{R}) \times \mathbb{R}^{2} ; \mathbb{R}\right)$ and there is a nonnegative constant $L$ such that for all $(x, y) \in \mathbb{R}_{+}, z, \bar{z} \in C(B, \mathbb{R}), p, \bar{p}, q, \bar{q} \in \mathbb{R}$, we have

$$
|f(x, y, z, p, q)-f(x, y, \bar{z}, \bar{p}, \bar{q})| \leq L\{\|z-\bar{z}\|+|p-\bar{p}|+|q-\bar{q}|\} ;
$$

(ii) $\phi \in C\left(\left[-a_{0}, \infty\right) \times\left[-b_{0}, \infty\right) \backslash(0, \infty)^{2} ; \mathbb{R}\right)$ and $\phi(0, \cdot) \in C^{1}\left(\mathbb{R}_{+} ; \mathbb{R}\right)$, $\phi(\cdot, 0) \in C^{1}\left(\mathbb{R}_{+}, \mathbb{R}\right) ;$

(iii) $h_{i} \in C^{1}\left(\left[-a_{0}, \infty\right) \times\left[-b_{0}, 0\right] ; \mathbb{R}\right), \quad i=1, \ldots, n, g_{j} \in C^{1}\left(\left[-a_{0}, 0\right] \times\right.$ $\left.\left[-b_{0}, \infty\right) ; \mathbb{R}\right), j=1, \ldots, m$, and

$$
h_{i}(x, y)=0, \quad g_{j}(x, y)=0, \quad(x, y) \in B, i=1, \ldots, n, j=1, \ldots, m ;
$$


(iv) there are nonnegative constants $M, N$ such that for all $(x, y) \in \mathbb{R}_{+}^{2}$, $i=1, \ldots, n, j=1, \ldots, m$, we have

$$
\begin{aligned}
& \left|h_{i}(x, y)\right| \leq N, \quad\left|D_{x} h_{i}(x, y)\right| \leq N, \\
& \left|g_{j}(x, y)\right| \leq M, \quad\left|D_{y} g_{j}(x, y)\right| \leq M ;
\end{aligned}
$$

(v) there is a positive constant $C$ such that

$$
\frac{L(2 C+1)}{C^{2}}+2 n N e^{C b_{n}}+2 m M e^{C a_{m}}<1 ;
$$

(vi) there are nonnegative constants $K_{1}, K_{2}$ such that

$$
\begin{gathered}
|\phi(x, 0)| \leq K_{1} e^{C x}, \quad\left|D_{x} \phi(x, 0)\right| \leq K_{1} e^{C x} \quad \text { for } x \in \mathbb{R}_{+} \\
|\phi(0, y)| \leq K_{1} e^{C y}, \quad\left|D_{y} \phi(0, y)\right| \leq K_{1} e^{C y} \quad \text { for } y \in \mathbb{R}_{+}, \\
|f(x, y, 0,0,0)| \leq K_{2} e^{C(x+y)} \quad \text { for }(x, y) \in \mathbb{R}_{+}
\end{gathered}
$$

Then there is a unique solution of the problem (1)-(3) in the class of functions $u \in U$ such that $\|u\|<\infty$.

Proof. Let $\widetilde{U}$ be the space of functions $u \in \widehat{U}$ such that $\|u\|_{C}<\infty$. We first prove that the operator $T$ defined by (8)-(10) maps $\widetilde{U}$ into itself. By the same argument as in the proof of Theorem 1 , if $u \in \widetilde{U}$ then $T u \in \widehat{U}$. It remains to show that $\|T u\|_{C}<\infty$. Note that

$$
\begin{aligned}
& (T u)(x, y) \\
& =\phi(x, 0)+\phi(0, y)-\phi(0,0) \\
& \quad-\sum_{i=1}^{n} h_{i}(x, 0) u\left(x, b_{i}\right)-\sum_{j=1}^{m} g_{j}(0, y) u\left(a_{j}, y\right) \\
& \quad+\int_{0}^{x y}\left[f\left(s, t, u_{(s, t)}, D_{x} u(s, t), D_{y} u(s, t)\right)-f(s, t, 0,0,0)\right] d t d s \\
& \quad+\int_{0}^{x} \int_{0}^{y} f(s, t, 0,0,0) d t d s \quad \text { for }(x, y) \in \mathbb{R}_{+}^{2},
\end{aligned}
$$

and hence, by (14)-(17), we get

$$
\begin{aligned}
|(T u)(x, y)| \leq & |\phi(x, 0)|+|\phi(0, y)|+|\phi(0,0)| \\
& +\sum_{i=1}^{n}\left|h_{i}(x, 0)\right| \cdot\left|u\left(x, b_{i}\right)\right|+\sum_{j=1}^{m}\left|g_{j}(0, y)\right| \cdot\left|u\left(a_{j}, y\right)\right| \\
& +L \int_{0}^{x} \int_{0}^{y}\left[\| u_{(s, t)}|+| D_{x} u(s, t)|+| D_{y} u(s, t) \mid\right] d t d s \\
& +\int_{0}^{x} \int_{0}^{y}|f(s, t, 0,0,0)| d t d s
\end{aligned}
$$




$$
\begin{aligned}
\leq & K_{1} e^{C x}+K_{1} e^{C y}+K_{1}+\sum_{i=1}^{n} N e^{C\left(b_{i}+x\right)}\|u\|_{C, 0} \\
& +\sum_{j=1}^{m} M e^{C\left(a_{j}+y\right)}\|u\|_{C, 0} \\
& +L \int_{0}^{x y}\left[\|u\|_{C, 0}+\|u\|_{C, 1}+\|u\|_{C, 2}\right] e^{C(s+t)} d t d s+\int_{0}^{x y} \int_{0}^{y} K_{2} e^{C(s+t)} d t d s \\
\leq & 3 K_{1} e^{C(x+y)}+\left[n N e^{C b_{n}}+m M e^{C a_{m}}\right]\|u\|_{C, 0} e^{C(x+y)} \\
& +\frac{1}{C^{2}}\left[L\|u\|_{C}+K_{2}\right] e^{C(x+y)} \quad \text { for }(x, y) \in \mathbb{R}_{+}^{2} .
\end{aligned}
$$

Since the above estimate also holds for $(x, y) \in\left[-a_{0}, \infty\right) \times\left[-b_{0}, \infty\right) \backslash(0, \infty)^{2}$ we see that

(19) $\|T u\|_{C, 0} \leq 3 K_{1}+\left[n N e^{C b_{n}}+m M e^{C a_{m}}\right]\|u\|_{C, 0}+\frac{1}{C^{2}}\left[L\|u\|_{C}+K_{2}\right]$.

Analogously, by (14)-(18), and by the estimates

$$
\begin{aligned}
\left|D_{x}(T u)(x, y)\right| \leq & \left|D_{x} \phi(x, 0)\right| \\
& +\sum_{i=1}^{n}\left|D_{x} h_{i}(x, 0)\right| \cdot\left|u\left(x, b_{i}\right)\right|+\sum_{i=1}^{n}\left|h_{i}(x, 0)\right| \cdot\left|D_{x} u\left(x, b_{i}\right)\right| \\
& +\int_{0}^{y}\left|f\left(x, t, u_{(x, t)}, D_{x} u(x, t), D_{y} u(x, t)\right)-f(x, t, 0,0,0)\right| d t \\
& +\int_{0}^{y}|f(x, t, 0,0,0)| d t \\
\leq & K_{1} e^{C x}+\sum_{i=1}^{n} N e^{C\left(b_{i}+x\right)}\left[\|u\|_{C, 0}+\|u\|_{C, 1}\right] \\
& +\int_{0}^{y}\left[\|u\|_{C, 0}+\|u\|_{C, 1}+\|u\|_{C, 2}\right] e^{C(x+t)} d t \\
& +\int_{0}^{y} K_{2} e^{C(x+t)} d t \\
\leq & K_{1} e^{C(x+y)}+n N e^{C b_{n}}\left[\|u\|_{C, 0}+\|u\|_{C, 1}\right] e^{C(x+y)} \\
& +\frac{1}{C}\left[L\|u\|_{C}+K_{2}\right] e^{C(x+y)}
\end{aligned}
$$

and

$$
\begin{aligned}
\left|D_{y}(T u)(x, y)\right| \leq & \left|D_{y} \phi(0, y)\right| \\
& +\sum_{j=1}^{m}\left|D_{y} g_{j}(0, y)\right| \cdot\left|u\left(a_{j}, y\right)\right|+\sum_{j=1}^{m}\left|g_{j}(0, y)\right| \cdot\left|D_{y} u\left(a_{j}, y\right)\right|
\end{aligned}
$$




$$
\begin{aligned}
& \quad+\int_{0}^{x}\left|f\left(s, y, u_{(s, y)}, D_{x} u(s, y), D_{y} u(s, y)\right)-f(x, t, 0,0,0)\right| d s \\
& \quad+\int_{0}^{x}|f(s, y, 0,0,0)| d s \\
& \leq K_{1} e^{C y}+\sum_{j=1}^{m} M e^{C\left(a_{j}+y\right)}\left[\|u\|_{C, 0}+\|u\|_{C, 2}\right] \\
& \quad+L \int_{0}^{x}\left[\|u\|_{C, 0}+\|u\|_{C, 1}+\|u\|_{C, 2}\right] e^{C(s+y)} d s+\int_{0}^{x} K_{2} e^{C(s+y)} d s \\
& \leq K_{1} e^{C(x+y)}+m M e^{C a_{m}}\left[\|u\|_{C, 0}+\|u\|_{C, 2}\right] e^{C(x+y)} \\
& \quad+\frac{1}{C}\left[L\|u\|_{C}+K_{2}\right] e^{C(x+y)},
\end{aligned}
$$

which hold for $(x, y) \in \mathbb{R}_{+}^{2}$, we get

$$
\begin{aligned}
& \|T u\|_{C, 1} \leq K_{1}+n N e C b_{n}\left[\|u\|_{C, 0}+\|u\|_{C, 1}\right]+\frac{1}{C}\left[L\|u\|_{C}+K_{2}\right], \\
& \|T u\|_{C, 2} \leq K_{1}+m M e C b_{m}\left[\|u\|_{C, 0}+\|u\|_{C, 2}\right]+\frac{1}{C}\left[L\|u\|_{C}+K_{2}\right] .
\end{aligned}
$$

Thus from (19)-(21) we obtain

$\|T u\|_{C} \leq 5 K_{1}+\frac{1+2 C}{C^{2}} K_{2}+\left\{\frac{L(1+2 C)}{C^{2}}+2 n N e^{C b_{n}}+2 m M e^{C a_{n}}\right\}\|u\|_{C}$,

which yields $\|T u\|_{C}<\infty$. Therefore, the operator $T$ maps $\widetilde{U}$ into itself.

As in the proof of Theorem 1 we show that $u \in U,\|u\|_{C}<\infty$, is a solution of the problem (1)-(3) if and only if it is a fixed point of the operator $T$. Repeating the coresponding computations from Theorem 1 we prove that $T$ is a contraction. Applying the Banach fixed point theorem completes the proof of Theorem 2 .

Remark 3. Theorem 2 remains valid with condition (iii) in a weaker form, analogous to that stated in Remark 2.

\section{References}

[1] L. Byszewski, Strong maximum and minimum principles for parabolic problems with nonlocal inequalities, Z. Angew. Math. Mech. 70 (1990), 202-205.

[2] - Existence and uniqueness of solutions of nonlocal problems for hyperbolic equation $u_{x t}=F\left(x, t, u, u_{x}\right)$, J. Appl. Math. Stochastic Anal. 3 (1990), 163-168.

[3] J. Chabrowski, On non-local problems for parabolic equations, Nagoya Math. J. 93 (1984), 109-131. 
[4] H. Chi, H. Poorkarmi, J. Wiener and S. M. Shah, On the exponential growth of solutions to nonlinear hyperbolic equations, Internat. J. Math. Math. Sci. 12 (1989), $539-546$

[5] T. Człapiński, Existence of solutions of the Darboux problem for partial differentialfunctional equations with infinite delay in a Banach space, Comment. Math. 35 (1995), 111-122.

[6] M. Krzyżański, Partial Differential Equations of Second Order, Vol. 2, Polish Sci. Publ., Warszawa, 1971.

[7] G. S. Ladde, V. Lakshmikantham and A. S. Vatsala, Monotone Iterative Techniques for Nonlinear Differential Equations, Pitman Adv. Publ. Program, Boston, 1985.

[8] W. Walter, Differential and Integral Inequalities, Springer, Berlin, 1970.

Institute of Mathematics

University of Gdańsk

Wita Stwosza 57

80-952 Gdańsk, Poland

E-mail: czltsz@ksinet.univ.gda.pl

Reçu par la Rédaction le 20.4.1995 